\title{
Influence of Synthesis Parameters in Obtaining KIT-6 Mesoporous Material
}

\author{
Fernando R. D. Fernandes, Francisco G. H. S. Pinto, Ewelanny L. F. Lima, Luiz D. Souza, \\ Vinícius P. S. Caldeira and Anne G. D. Santos *
}

Laboratory of Catalysis, Environment and Materials, Department of Chemistry, State University of Rio Grande do Norte, Mossoró/RN 59610-090, Brazil; fe.rodrigo@hotmail.com (F.R.D.F); gustavo_sk13@hotmail.com (F.G.H.S.P.); ewelannylouyde@hotmail.com (E.L.F.L.); luizsouza@uern.br (L.D.S.); viniciuscaldeira@uern.br (V.P.S.C.)

* Correspondence: annegabriella@uern.br; Tel.: +55-084-3315-2241

Received: 16 April 2018; Accepted: 30 April 2018; Published: 5 May 2018

\begin{abstract}
In the present work, modifications were made in the typical synthesis of KIT-6 mesoporous material. The molar ratio of P123 and its dissolution time, the type of alcohol, the aging time, and the heat treatment time were varied. The materials obtained were characterized by $\mathrm{X}$-ray diffraction (XRD), thermogravimetry and differential thermogravimetry (TG/DTG), Fourier-transform infrared spectroscopy (FTIR), adsorption and desorption of $\mathrm{N}_{2}$ and transmission electron microscopy (TEM). It was observed that the modifications interfered directly in the ordered structure of materials, displaying materials with cubic structures Ia3d (KIT-6 mesoporous material) and hexagonal structures P6mm, (SBA-15 mesoporous material). The type of alcohol probably acts on generation of the micelle, influencing the formation of the porous system and ordered structure. The results obtained indicate that the cubic structure of KIT- 6 can be formed with a reduction of $30 \%$ in the P123 concentration, and decreases of 2, 6 and $12 \mathrm{~h}$ in times of P123 dissolution, aging and heat treatment, respectively. The modifications carried out in the synthesis procedure have resulted in ir being possible to employ materials with different characteristics, such as mesoscopic ordering and textural properties, in applications in the areas of catalysis and adsorption.
\end{abstract}

Keywords: mesoporous silica; KIT-6; synthesis parameters; cubic structure

\section{Introduction}

Mesoporous materials have been gaining considerable attention within the field of catalysis, adsorption and ion exchange, due to their unique properties and diverse applicability [1]. The KIT- 6 mesoporous material has been intensely investigated by academics and industries in the last few years [2]. It has this name for having been discovered in the Korea Institute of Science and Technology [3]. It has a large average pore diameter (4-12 nm), wall thickness of 4 to $6 \mathrm{~nm}$, and a tridimensional symmetric cubic structure Ia3d $[4,5]$. It also has a large pore volume, a high surface area and a bi continuous interpenetrating network, with additional micropores [2,6,7]. The pores of the KIT- 6 provide easy and direct access to the active sites, which facilitates the insertion or diffusion of species in its interior [8].

The synthesis of KIT-6 was reported by Kleitz et al. [9]. In a typical hydrothermal synthesis of this material, hydrochloric acid is used to keep the medium acid, the triblock copolymer P123 as organic structure template, tetraethylorthosilicate (TEOS) as a source of silica; by contrast with materials such as SBA-15, butanol is used as a co-solvent and co-template. Each reagent has an indispensable function in the synthesis of KIT-6. The template enables the creation of materials with pores of different sizes and defined morphologies, thus producing channels that permeate the material [10]. 
The source of silica has the function of being the base material that will form the walls of the porous structure. In a typical synthesis of KIT-6, tetraethylorthosilicate (TEOS) is used due to its low cost and toxicity. The use of alcohol has the function of influencing the behavior of micelles during the synthesis process [11], facilitating P123 dissolution and helping in the organization of the cubic structure. The typical synthesis of KIT- 6 follows pre-established times, which are necessary for the formation of its structure. Although there are no reports of works about the modification of reagents and stages of the KIT-6 synthesis process, there are several works about modifications in these parameters for similar mesoporous materials [12-15]. However, studies aimed at reducing the synthesis time for this material are relevant because, as seen by Impéror-Clerc et al. [12] in a study about SBA-15, the micelles of the template (P123) are already present in the first minutes of the synthesis.

Various studies have been developed with materials similar to KIT- 6 with the goal of modifying parameters and reagents in order to evaluate the behavior of such variations in the final material. In a study performed by Cao et al. (2009) [15], the authors substituted P123 in the synthesis of SBA-15 for copolymers of polyethylene oxide (EO) and methyl polyacrylate (MA), which showed good results, promoting a material with hexagonal structure and good pore size distribution. Modifications in the source of the silica, as well as in the formation and organization stages of these materials, are also documented in the literature [14,16-18]. Studies demonstrating modifications in the synthesis parameters and obtaining materials with different properties are also reported for other types of materials, such as ordered mesoporous carbon (OMC) $[19,20]$. Such studies show the importance of analyzing the synthesis parameters and how they interfere in the final material.

Considering the promising characteristics of KIT-6 mesoporous material and due to the fact that there are no reports about modifications of its synthesis, the present study aims to vary parameters of KIT-6 synthesis in order to evaluate these changes in the structure and properties of the material.

\section{Materials and Methods}

\subsection{Synthesis of Standard KIT-6}

The standard KIT-6 sample was obtained following the typical method described by Kleitz et al. [9], using the following molar ratio: 1.000 TEOS; $0.017 \mathrm{P} 123 ; 1.83 \mathrm{HCl} ; 195 \mathrm{H}_{2} \mathrm{O} ; 1.31$ butanol. For $0.05 \mathrm{~kg}$ of synthesis gel, $1.23 \mathrm{~g}$ of triblock copolymer pluronic (P123, $\mathrm{EO}_{20}-\mathrm{PO}_{70}-\mathrm{EO}_{20}$, Sigma Aldrich, St. Louis, MI, USA) was dissolved in $2.25 \mathrm{~g}$ of hydrochloric acid ( $37 \%$ Vetec) and $44 \mathrm{~g}$ of distilled water, this solution was subjected to vigorous stirring at $308 \mathrm{~K}$ for $6 \mathrm{~h}$. After complete dissolution, $1.21 \mathrm{~g}$ butanol $(99.4 \%$ Vetec) was added. The mixture remained under stirring at $308 \mathrm{~K}$ for $1 \mathrm{~h}$. Thereafter, the tetraethylorthosilicate (TEOS, 98\% Sigma Aldrich, St. Louis, MI, USA) was added to the homogeneous solution. Then, this mixture was kept under stirring at $308 \mathrm{~K}$ for $24 \mathrm{~h}$, and in sequence was transferred to an autoclave and heated at $373 \mathrm{~K}$ for $24 \mathrm{~h}$ under static conditions. The solid product finally obtained was separated by vacuum filtration, washed with $2 \% \mathrm{HCl} /$ ethanol solution, and dried overnight at room temperature. Subsequently, the material was calcined at $823 \mathrm{~K}$ for $6 \mathrm{~h}$ in synthetic air atmosphere to remove the organic template. The sample obtained through the standard synthesis was coded as KIT-6.

\subsection{Modifications in the Standard Synthesis}

The changes in the parameters of the synthesis of KIT- 6 were carried out starting from the synthesis adopted as standard [9], altering only one parameter at a time, keeping the others unchanged.

The molar ratio of organic template P123 was modified by changing the standard ratio of 0.017 to 0.012 and 0.022 . Samples obtained through this procedure were coded as K0.012 and K0.022, respectively. The dissolution time of the organic template was systematically modified, altering the initial time from $6 \mathrm{~h}$ to 8,4 and $2 \mathrm{~h}$, coded by KDT8, KDT4 and KDT2, respectively. Another modified parameter was the standard alcohol (butanol), which was replaced by the following alcohols: methanol (Synth 99.4\%), ethanol (Synth 99.5\%), 1-propanol (99.5\% Vetec) and 1-pentanol (99\% Sigma Aldrich). 
These samples were coded as KMt, KEt, KPr and KPe, respectively. The aging time of the synthesis gel was also modified, varying the standard time from $24 \mathrm{~h}$ to $6,12,18$ and 30, with KAT6, KAT12, KAT18 and KAT30 samples, respectively. The last modified parameter was the time of heat treatment, changing the initial time from 24 to 12,18 and $30 \mathrm{~h}$, which were coded as KHT 12, KHT 18 and KHT 30, respectively.

Figure 1 shows a representative scheme with a summary of all modified parameters in the KIT-6 synthesis, and the respective sample codes for each parameter.

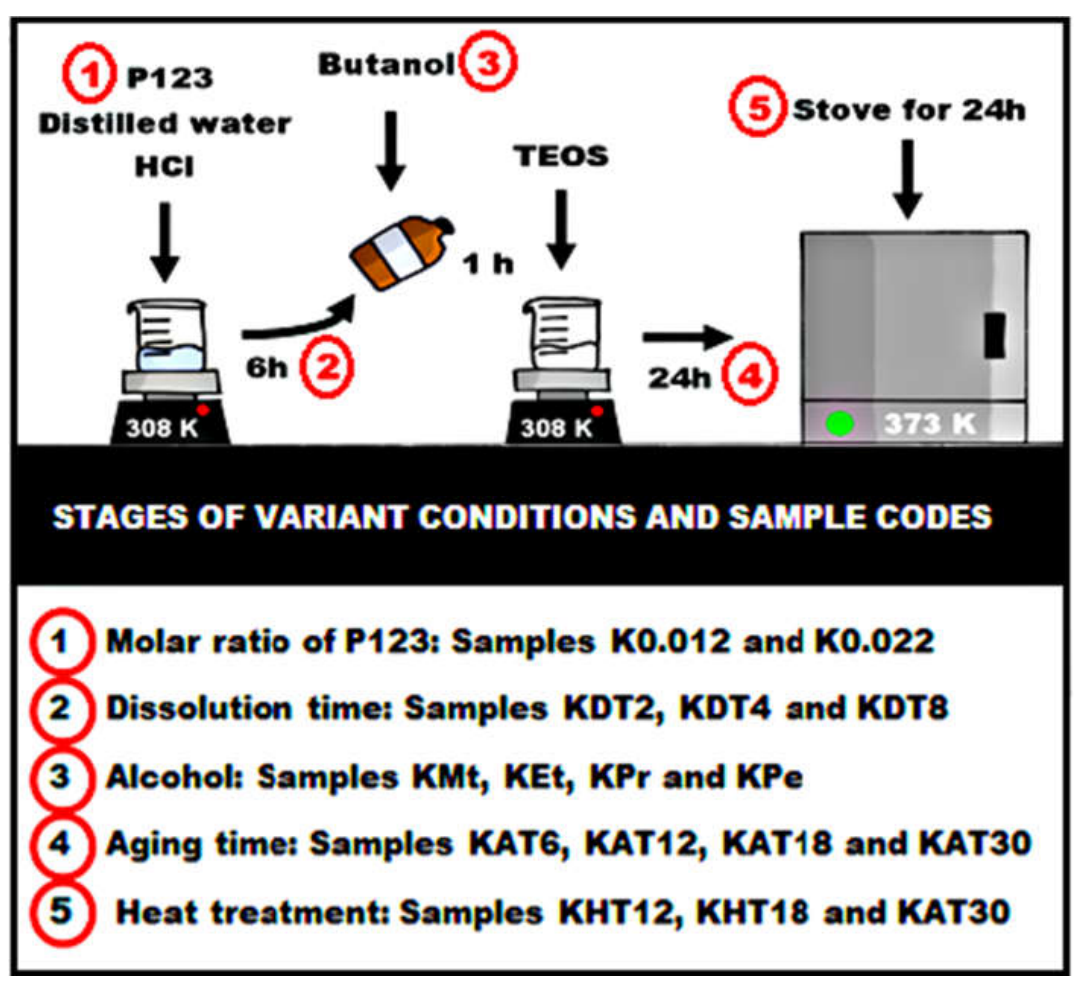

Figure 1. Schematic of the modifications made in the KIT-6 synthesis.

\subsection{Characterizations}

The X-ray diffraction (XRD) patterns were carried out on a Rigaku Mini Flex II equipment using $\mathrm{CuK} \alpha$ radiation at a voltage of $30 \mathrm{KV}$ and a $15 \mathrm{~mA}$ tube current. The data were collected at low-angle $0.5-3.0^{\circ} 2 \theta$, with step of $0.005^{\circ}$ and time of $0.4 \mathrm{~s}$. Thermogravimetric analysis (TGA) was performed on the STA 449F3-Jupiter equipment. About $5 \mathrm{mg}$ of material was used in an alumina crucible under heating from $298-1173 \mathrm{~K}$, with a heating rate of $10 \mathrm{~K} \cdot \mathrm{min}^{-1}$, by a dynamic nitrogen atmosphere with flow of $25 \mathrm{~mL} \cdot \mathrm{min}^{-1}$. The experiments of adsorption and desorption of $\mathrm{N}_{2}$ were performed at $77 \mathrm{~K}$ on ASAP 2020 Micromeritics equipment. Prior to measurement, the samples were degassed at $573 \mathrm{~K}$ for $10 \mathrm{~h}$ under vacuum. The Brumauer-Emmett-Teller (BET) method was used to calculate the specific area [21] using the Rouquerol criterion to better apply this method [22]. The mesoporous volume was determined from the $\alpha$-plot method for ordered mesoporous materials ( $\alpha$-plot OMM), and the total volume obtained by the Gurvich method [23]. The pore size distributions (PSD) were derived from the adsorption branch of the isotherms using the methods of Barrett-Joyner-Halanda (BJH), Villarroel-Bezerra-Sapag (VBS) [24] and the non-local density functional theory (NLDFT) [25]. For discussion of pore diameter, the VBS method was also applied in the desorption branch. Fourier-transform infrared spectroscopy (FTIR) spectra were obtained in the medium infrared region of $4000-400 \mathrm{~cm}^{-1}$ with $4 \mathrm{~cm}^{-1}$ resolution in a Bomem spectrophotometer model MB102. Transmission electron microscopy (TEM) was performed on Jeol microscope equipment, model JEM-2100 CM-200 (200 KV). Before analysis, the sample was 
dispersed in ethanol using low frequency ultrasound and placed in a copper screen coated with carbon. Through the TEM images it was possible to determine the pore diameter for KDT4 and KHT12 samples. The values were measured from the ImageJ software, using the arithmetic mean for 50 points, with a standard deviation less than $20 \%$.

\section{Results and Discussions}

Figure 2 shows the low-angle diffractograms of all the synthetized samples.
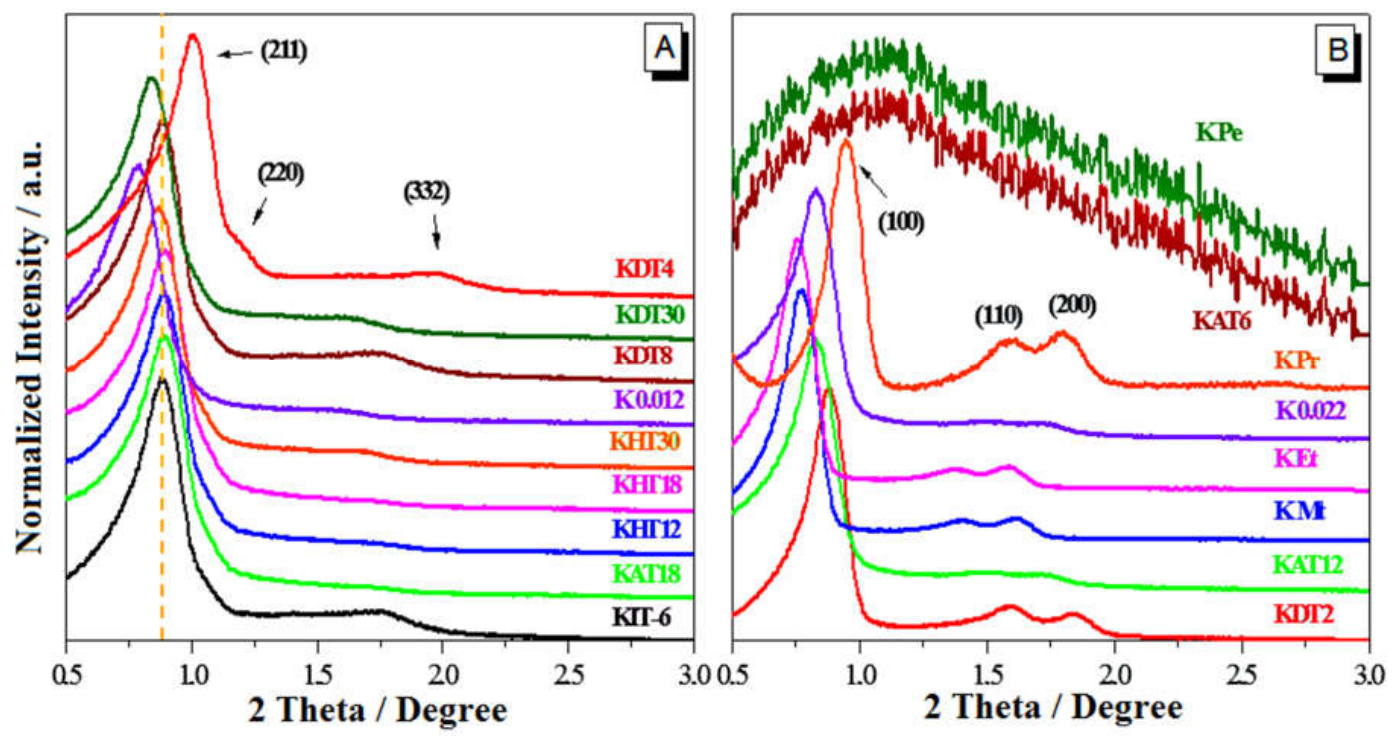

Figure 2. X-ray diffraction (XRD) patterns of all synthesized samples.

The samples in Figure 2A show reflection characteristics of materials with KIT-6 type, displaying (211), (220) and (332) Miller indices that are characteristic of three-dimensional mesoporous structures with spatial group Ia3d, typical of structures organized in cubic array $[9,26,27]$. The samples K0.022, KDT2, KMt, KEt, KPr and KAT12 in Figure 2B show reflections with (100), (110) and (120) Miller indices. According to Zhao et al. [28], these reflections are characteristic of two-dimensional mesoporous structures with spatial group P6mm, typical of structures organized in a hexagonal array, corresponding to SBA-15 type materials. The samples KAT6 and K Pe in Figure 2B not show any arranged structure.

Based on the data of the modification in the molar proportion of the organic template, two different behaviors can be noticed. The sample synthetized with a lower quantity of template (K0.012 sample) showed similar results to the standard KIT-6. This demonstrates that, even with the reduction employed in the synthesis, there was no interference in the critical micellar concentration (CMC) and subsequent formation of 3D cubic structure desired. A different behavior was observed with regard to the sample with a higher than standard molar proportion (K0.022 sample). The formation of a 2D hexagonal structure was noticed, referent to the SBA-15 mesoporous material. According to Wanka et al. [29], the increase in the quantity of the template used in the synthesis at constant temperature can lead to a transition in the cubic phase to the hexagonal phase, as can be observed in the present study. Figure 3A shows a representative scheme of the data obtained in this modification stage.

For the modification performed at the dissolution time of the organic template, it is noticeable that, by reducing the standard time from $6 \mathrm{~h}$ to $2 \mathrm{~h}$, a cubic Ia3d structure is not obtained. However, for this reduction time a SBA-15 material with high P6mm hexagonal organization is obtained. This behavior can be related to the micellar formation and posterior protonation of the EO (ethylene oxide) groups in the early synthesis stage. The short period of $2 \mathrm{~h}$, possibly, is not enough for the interaction of the micelles with the inorganic phase of silica, thus favoring the formation of a two-dimensional structure, which is thermodynamically more favorable than a three-dimensional one. For all the samples with 
times higher than $2 \mathrm{~h}$, the formation of cubic structure-like KIT-6 material was observed (Figure 3B). However, when observing the XRD peaks of the KDT4 sample, a displacement to a higher value of $2 \theta /$ angle in comparison to the KIT- 6 standard was noted. This behavior demonstrates an interference in the structural organization of the material. Such results determine that the required time to the dissolution of the P123 template directly influences the formation and structural organization in the synthesis of the KIT-6 material.

The modifications made in the type of alcohol employed in the standard synthesis showed significant data regarding the study of the synthesis mechanism of ordered mesoporous material. It was possible to observe that all alcohols with carbonic chains shorter than butanol (KMt, KEt and KPr samples) exhibited a structure of SBA-15 type, while the alcohol with the higher carbonic chain, 1-pentanol (sample KPe), did not display any ordered structure. In a study developed by Kim et al. [30], the authors proposed that butanol interacts with the chains of PEO (polyethylene oxide) and PPO (polypropylene oxide) of the P123 micelles behaving as a type of co-template. Thus, the butanol alcohol acts directly on the curvature of the bi-continuous channels and is responsible for forming the cubic structure of the KIT- 6 material. Considering this study and the results obtained in the present work, it has been shown that the type of alcohol is an important parameter for the generation of the Ia3d cubic structure. Due to the small size of the carbonic chains (methanol, ethanol and propanol), the interaction of the steric forces exerted by these alcohols in the micelle is possibly reduced. In this case, the smaller carbon chains are probably not enough to promote the posterior curvature of channels, resulting in unidirectional channels typical of SBA-15 materials. An opposite behavior was observed when increasing the size of the carbonic chain (KPe sample). The data probably suggest that pentanol alcohol provides an excessive interaction between the micelles to the point of promotion of the collapse of the structure. Figure $3 \mathrm{C}$ shows a representative scheme as a proposal of the influence of the variation on the type of alcohol in the formation mechanism of the KIT-6 material.

The modifications on the aging time of the synthesis gel showed that, with the increase of time, the formation of the cubic structure is favored. This behavior reveals that $6 \mathrm{~h}$ (KAT6 sample) is inadequate to the formation of any organized structure. This fact can be related to the low rate of hydrolysis and posterior condensation of the silica groups. From $12 \mathrm{~h}$ (KAT12 sample) of aging time the formation of a material with hexagonal phase and unidirectional channels like the SBA-15 material was noticed. This fact corroborates the results described by Kim et al. [30], where the authors observed the formation of a unidirectional phase with low synthesis time, which preceded the cubic phase. In addition to the present discussion, some studies showed that the change in synthesis parameters could cause a transition from the hexagonal to the cubic phase, and later to a lamellar phase [31,32]. Corroborating these studies, this study demonstrated that the $\mathrm{X}$-ray diffraction behavior at longer aging times, such as $18 \mathrm{~h}$ and $30 \mathrm{~h}$ (KAT18 and KAT 30 samples), provided the formation of the Ia3d cubic structure characteristic of KIT-6 material. This case reveals that the "organic-inorganic" agglomerate obtained by the deposition of silicate species over the co-polymer needs periods longer than $18 \mathrm{~h}$ to go from a two-dimensional hexagonal organization with one-directional channels to a cubic three-dimensional phase with curved channels. Another relevant discussion refers to the curvature of the bi-continuous channels of the KIT- 6 material. This indicates that the adequate time for there to be interaction forces exercised by the alcohol in the P123 micelles is between $12 \mathrm{~h}$ and $18 \mathrm{~h}$ of aging time in the synthesis gel. Considering this fact, even when using the standard alcohol for all the syntheses of this modification stage, only with times higher than $18 \mathrm{~h}$ can the desired Ia3d cubic structure be obtained (see scheme in Figure 3D).

The results of the variation on the thermal treatment time of synthesis gel show that all of the materials of this modification stage (KHT12, KHT18 and KHT30 samples) had structure typical of KIT-6, XRD peaks that were very similar to the standard sample. This fact indicates that all of the formation stages and structural organization of the cubic phase are probably found in the earlier stages of the synthesis mechanism. Thus, it is possible to obtain a high degree of condensation of the $\mathrm{Si}-\mathrm{OH}$ groups and later formation of Si-O-Si bonds without any significant interference in the material's 
structure with a reduction of up to $12 \mathrm{~h}$ of the standard time. It should be noted that modifications performed in this parameter of the synthesis demonstrated greater influence on the textural properties (discussions performed posteriorly).
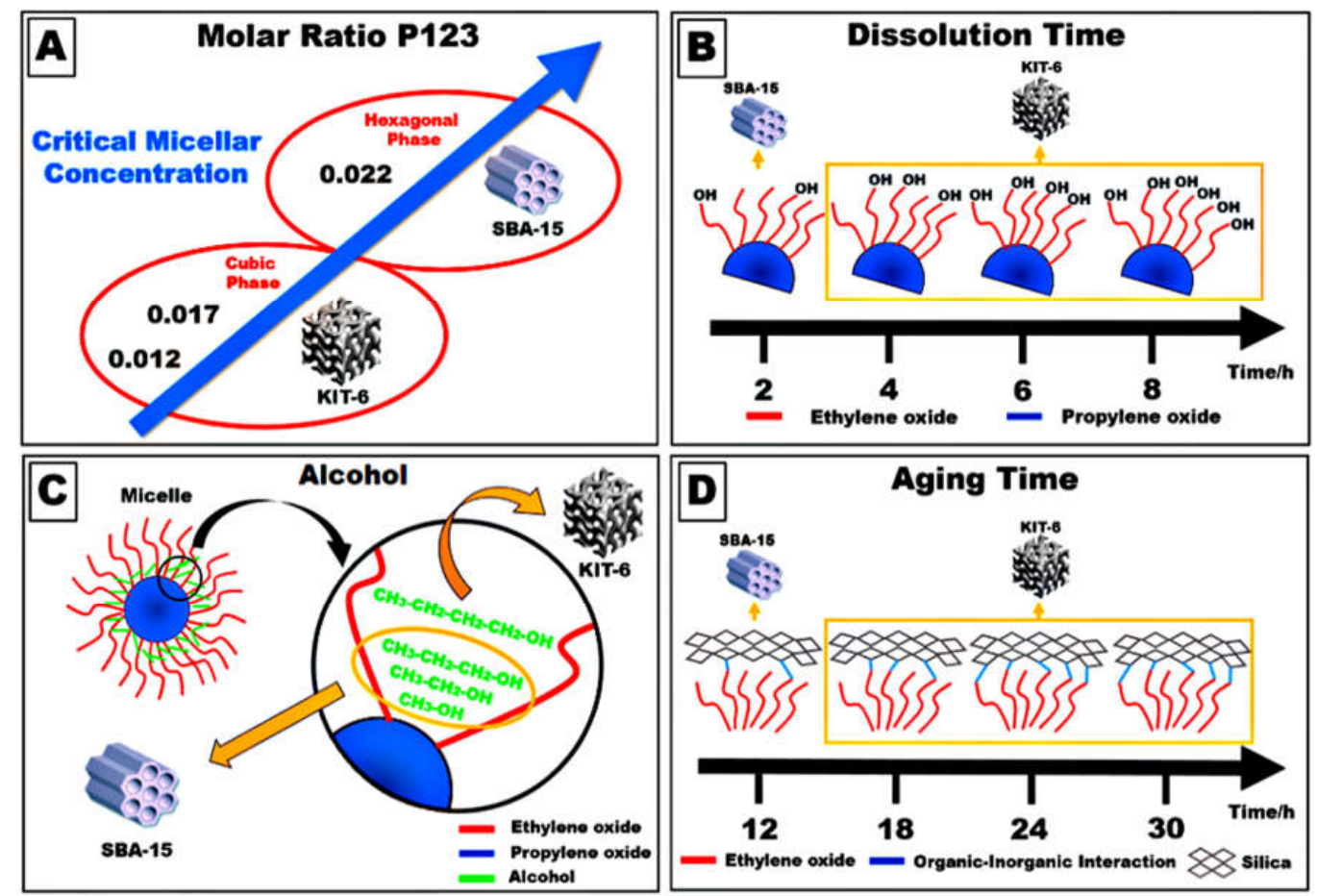

Figure 3. Representative scheme of the data obtained in: (A) the molar ratio of P123; (B) P123 dissolution time; (C) alcohol; and (D) aging time.

By the XRD data, the interplanar distance $\left(\mathrm{d}_{(211)}\right.$ and $\left.\mathrm{d}_{(100)}\right)$, and the mesoporous parameters $\left(\mathrm{a}_{\mathrm{cub}}\right.$ and $a_{\text {hex }}$ ), for cubic and hexagonal structures, respectively, could also be determined. Table 1 exhibit these values for all the samples. In general, the samples showed cubic structures had very similar behavior to the standard KIT-6, except in some specific cases discussed below.

Table 1. Interplanar distance and mesoporous parameter of all samples with ordered structures.

\begin{tabular}{ccccc}
\hline Samples & $\mathbf{d}_{(\mathbf{2 1 1})} / \mathbf{n m}$ & $\mathbf{d}_{(\mathbf{1 0 0})} / \mathbf{n m}$ & $\mathbf{a}_{\mathbf{C u b}} / \mathbf{n m}$ & $\mathbf{a}_{\mathbf{H e x}} / \mathbf{n m}$ \\
\hline KIT-6 & 5.14 & - & 12.60 & - \\
K0.012 & 5.50 & - & 13.50 & - \\
K0.022 & & 10.70 & - & 12.37 \\
KDT2 & & 11.00 & - & 12.70 \\
KDT4 & 4.53 & - & 11.10 & - \\
KDT8 & 5.15 & - & 12.60 & - \\
KMt & & 11.00 & - & 12.70 \\
KEt & & 11.40 & - & 13.17 \\
KPr & & 10.10 & - & 11.67 \\
KAT12 & & 10.80 & - & 12.30 \\
KAT18 & 5.12 & - & 12.54 & - \\
KAT30 & 5.20 & - & 12.92 & - \\
KHT12 & 5.13 & - & 12.56 & - \\
KHT18 & 5.14 & - & 12.60 & - \\
KHT30 & 5.17 & - & 12.66 & - \\
\hline
\end{tabular}

$\mathrm{d}_{(211)}=$ interplanar distance calculated from $\lambda \mathrm{CuK} \alpha=2 \mathrm{~d}_{(211)}$. $\operatorname{sen} \theta ; \mathrm{d}_{(100)}=$ interplanar distance calculated from $\lambda \mathrm{CuK} \alpha=2 \mathrm{~d}_{(100)}$. sen $\theta ; \mathrm{a}_{\mathrm{Cub}}=$ cubic parameter calculated from $\mathrm{a}_{\mathrm{Cub}}=\mathrm{d}_{(211)} \cdot 6^{1 / 2}$; $\mathrm{a}_{\mathrm{Hex}}=$ hexagonal parameter calculated from $\mathrm{a}_{\mathrm{Hex}}=2 \mathrm{~d}_{(100)} / 3^{1 / 2}$. 
The K0.012 and KAT30 samples presented interplanar distance and mesoporous parameter values higher than the standard KIT-6, while the sample KDT4 presented lower values. Relative to the decrease in P123 concentration (K0.012 sample), it can be determined that this variation directly interfered in the organization of the channels, increasing the distance between them. The data obtained for the KAT30 sample shows that the increase in the standard aging time interferes in the organization of the channels formed in the material. This fact can be related to the function of alcohol in the KIT-6 synthesis, which at long times can lead to an excessive curvature and consequently an influence in the structure, with $24 \mathrm{~h}$ being the ideal time. The behavior observed in the KDT4 sample denotes that the decrease in the time destined for the micellar formation directly modifies the organization of the future channels, generating significant reduction of $1.5 \mathrm{~nm}$ of the mesoporous parameter.

Even for the samples that had two-dimensional hexagonal structures, interesting behaviors can be observed. Analyzing the samples obtained from the alcohol modification, an increase on the values of $d_{(100)}$ and $a_{\text {hex }}$ of the KMt compared to KEt samples can be observed, and a decrease on these values for KPr sample can be seen. This behavior demonstrates that the alcohol probably can act as a co-solvent or co-template, having a greater influence on the structure according to the size of its chain. Thus, alcohols with chains (C1-C2) that are too short do not interfere in a significant way on the hexagonal organization, indicating the behavior of a co-solvent. However, alcohols with longer chains interfere on the structural organization (the propanol (C3) in this case) or act in such a way that it completely modifies the structure of the material, except for the butanol (C4) that acts as a co-template.

The thermogravimetry and differential thermogravimetry (TG/DTG) curves and FTIR spectra for KIT-6, K0.012 and KDT4 representative samples has been represented in Figure 4A-C. The data of other samples can be seen in the supplementary material (Figures S1-S3).
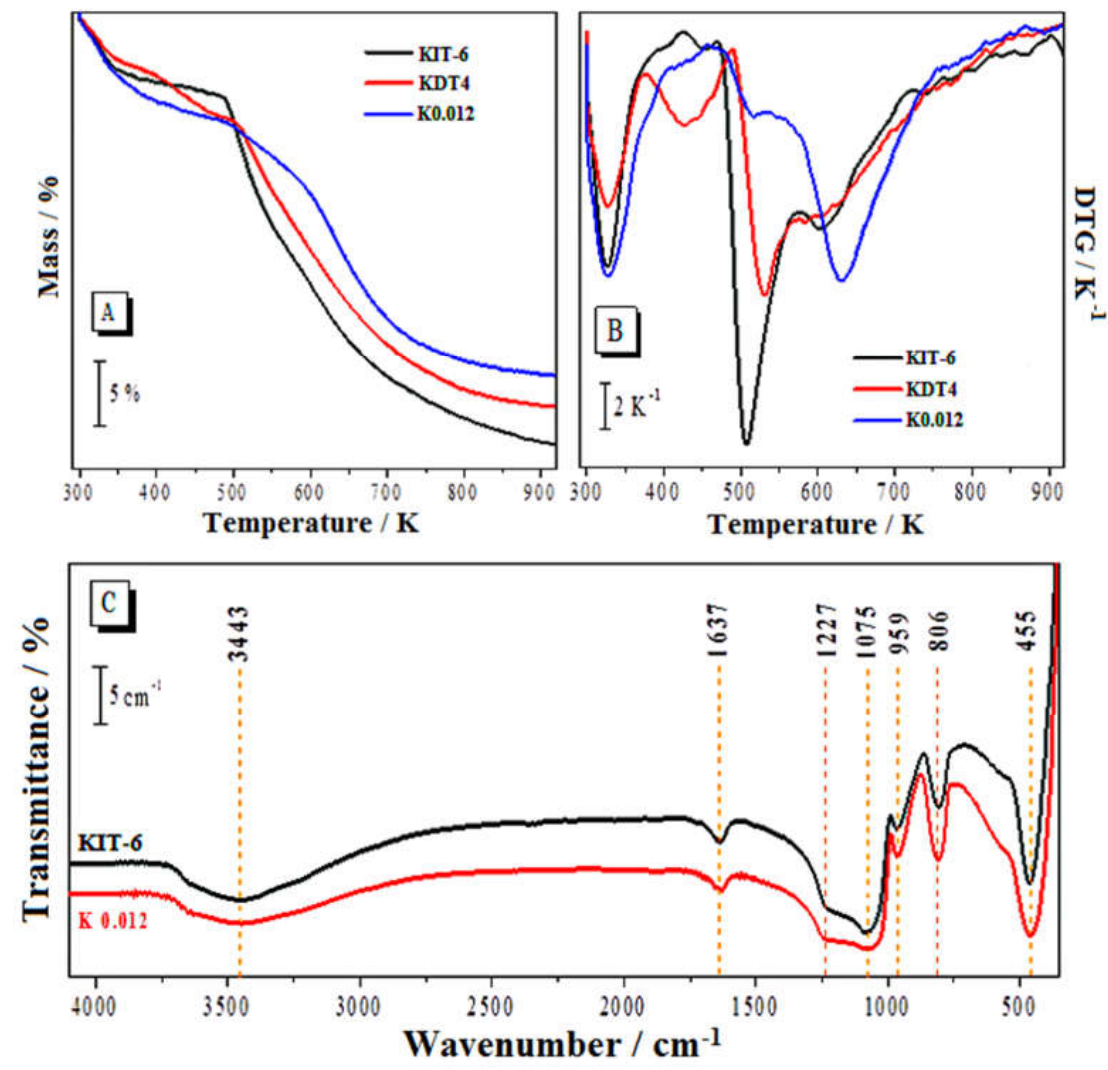

Figure 4. (A) Thermogravimetry (TG) and (B) differential thermogravimetry (DTG) curves of the KIT-6, K0.012 and KDT4 samples; and (C) the Fourier-transform infrared spectroscopy (FTIR) spectra of the standard KIT-6 and KDT2 samples. 
In general, it can be observed that all samples had between two or more mass losses, according the Table 2 .

Table 2. Quantification of mass loss events of all samples with ordered structures.

\begin{tabular}{ccccccccc}
\hline \multirow{2}{*}{ Samples } & \multicolumn{4}{c}{ Events/K } & \multicolumn{5}{c}{ Mass Loss/\% } \\
\cline { 2 - 9 } & I & II & III & IV & I & II & III & IV \\
\hline KIT-6 & $298-418$ & $418-583$ & $583-823$ & - & 5.6 & 14.6 & 10.4 & - \\
K0.012 & $298-443$ & $443-538$ & $538-823$ & & 7.7 & 2.6 & 16.6 & \\
K0.022 & $298-383$ & $383-546$ & $546-823$ & & 12.2 & 5.0 & 15.3 & - \\
KDT2 & $298-383$ & $383-565$ & $565-823$ & - & 4.4 & 24.0 & 14.6 & 10.5 \\
KDT4 & $298-375$ & $375-489$ & $489-588$ & $588-823$ & 4.2 & 4.0 & 9.8 & \\
KDT8 & $298-460$ & $460-600$ & & & 4.6 & 14.6 & & - \\
KAT12 & $298-388$ & $388-598$ & $598-823$ & - & 10.0 & 17.6 & 4.0 & - \\
KAT18 & $298-463$ & $463-823$ & - & - & 10.2 & 14.7 & - & - \\
KAT30 & $298-500$ & $418-610$ & & & 4.6 & 16.6 & & \\
KMt & $298-403$ & $403-603$ & $603-823$ & & 11.6 & 14.9 & 5.0 & \\
KEt & $298-398$ & $398-583$ & $583-823$ & & 9.8 & 18.0 & 4.3 & \\
KPr & $298-413$ & $413-593$ & $593-823$ & & 13.4 & 13.0 & 6.4 & - \\
KHT12 & $298-458$ & $458-823$ & - & - & 6.2 & 17.1 & - & - \\
KHT18 & $298-458$ & $458-823$ & - & - & 9.0 & 17.4 & - & - \\
KHT30 & $298-459$ & $459-823$ & - & - & 10.3 & 15.0 & - & - \\
\hline
\end{tabular}

It can be observed that the standard KIT-6 showed three well-defined mass losses. The first loss corresponds to the removal of physisorbed water in the interior of the materials; the second loss is related to the degradation of the organic template and the removal of chemisorbed water; the third loss corresponds to the condensation of the remaining silanol groups in the surface and in the pores of the materials $[33,34]$. In general, the other samples showed similar losses, with some cases of specific changes in the temperature intervals of the mass losses and the quantity of losses being also observed. One of these cases was the KDT4 sample, in which four mass losses were observed. This higher difficulty on the removal of the organic template can be directly connected to the disorder found in its structure, which corroborates with the XRD results. Another pertinent discussion is in regard to the samples that formed hexagonal structures with unidirectional channels of the SBA-15 type, according to the XRD technique. All samples showed the second mass loss event at lower initial temperatures compared to the other samples with KIT-6 structure (see Figure S2 in the Supplementary Material). This fact can demonstrate a greater facility on the removal of the organic template due to the unidirectional system of its channels.

Figure 4C presents the FTIR spectra of the standard KIT-6 and the KDT4 and KDT2 representative samples for the ordered 3D cubic and unidirectional hexagonal structures. In general, bands related to the stretches of the inorganic functional groups, in both KIT-6 and SBA-15 materials, can be observed. In all samples were identified the absorption bands around 3443, 1637, 1227, 1075, 959, 806 and $455 \mathrm{~cm}^{-1}$.

The band around $3443 \mathrm{~cm}^{-1}$ is attributed to the stretching vibrations of the hydroxyl groups, related to the $\mathrm{O}-\mathrm{H}$ of the $\mathrm{Si}-\mathrm{OH}$ groups found in the samples [35]. The band around $1637 \mathrm{~cm}^{-1}$ is attributed to the interaction of water with the support surface [36]. Bands around $1075 \mathrm{~cm}^{-1}$ and the identified shoulder at $1227 \mathrm{~cm}^{-1}$ are typical bands referring to asymmetric stretches of the Si-O-Si bonds. The band an $806 \mathrm{~cm}^{-1}$ are attributed to symmetrical stretches of the Si-O-Si bonds, and the bands near 959 and $455 \mathrm{~cm}^{-1}$ refer respectively to the symmetrical and asymmetric stretches of the $\mathrm{Si}-\mathrm{O}$ bonds of the $\mathrm{Si}-\mathrm{OH}$ groups [37].

The structural profile mentioned above was observed for both samples with 3D cubic structure and 2D hexagonal structure. This fact occurs because the materials have in their composition only bonds between $\mathrm{Si}$ and $\mathrm{O}$ atoms, including the formation of silanols ( $\mathrm{Si}-\mathrm{OH})$ groups.

Figure 5 shows the results of adsorption and desorption analysis of $\mathrm{N}_{2}$ at $77 \mathrm{~K}$, performed on samples that presented ordered structures. 

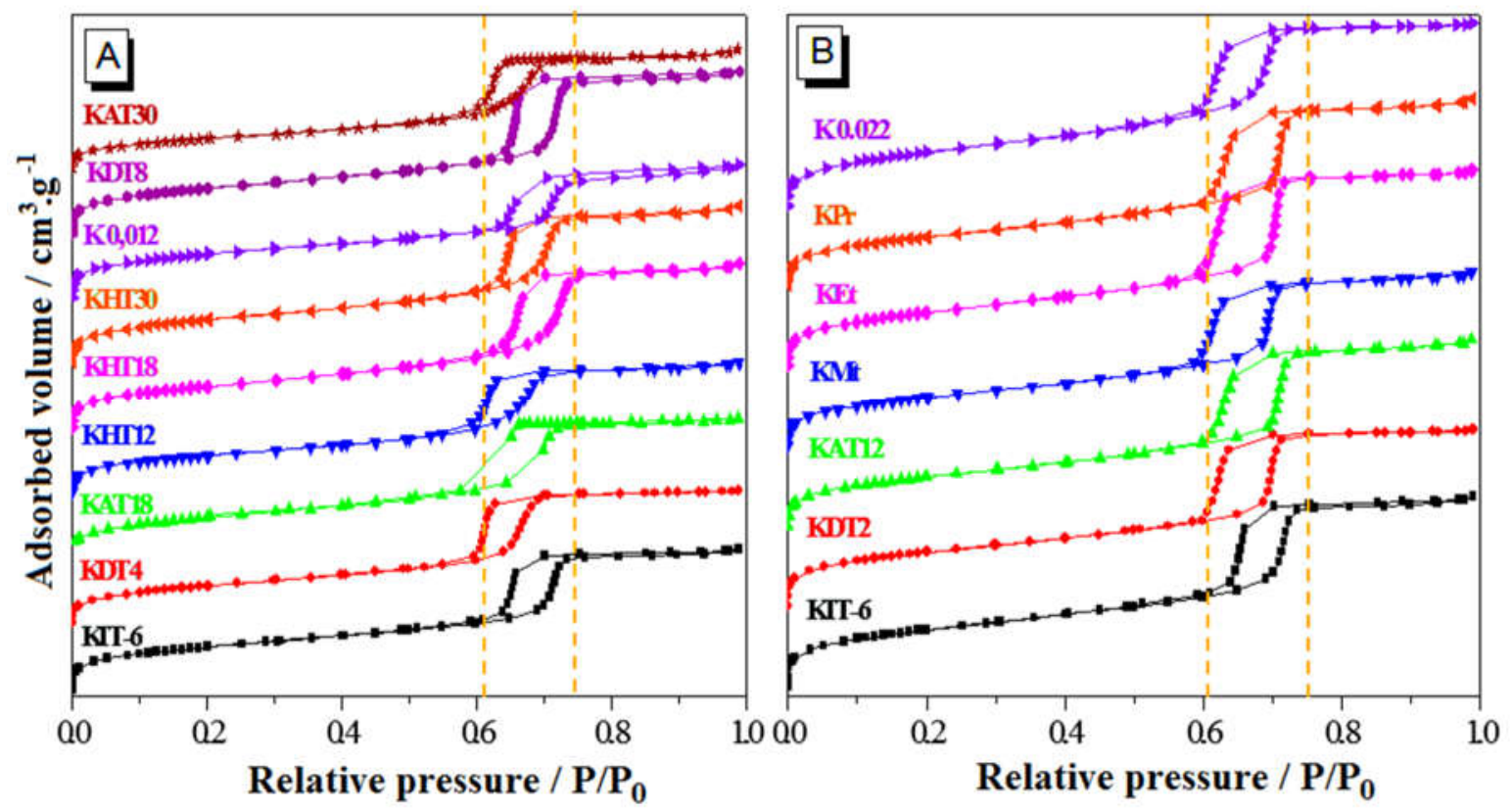

Figure 5. $\mathrm{N}_{2}$ adsorption and desorption isotherms of the (A) samples that presented cubic structures and (B) samples that presented hexagonal structures.

From the isotherms, it can be observed that regardless of the modifications made during the synthesis procedure, all of the samples exhibited isotherms classified as type IV (a), characteristic of mesoporous materials with capillary condensation between 0.6 and $0.8 \mathrm{P} / \mathrm{P}_{0}$, and a typical final saturation plateau [38]. As well, the referred capillary condensation was accompanied by H1 hysteresis loop. This hysteresis loop is characteristic of materials that show a cylindrical pores system [38]. Furthermore, it is relevant to highlight that all of the materials showed a very narrow hysteresis loops (between 0.6 and $0.75 \mathrm{P} / \mathrm{P}_{0}$ ), which indicates a pores system with high uniformity. The exception was the KAT18 sample that exhibited a small loss of verticality in the hysteresis, which can be attributed to a porous system with higher distribution rate of average pore sizes.

Table 3 presents the textural properties of all samples with ordered structure.

Table 3. Textural properties of samples with ordered structures.

\begin{tabular}{|c|c|c|c|c|}
\hline Samples & $\mathrm{S}_{\mathrm{BET}} / \mathrm{m}^{2} \cdot \mathrm{g}^{-1}$ & $\mathrm{~V}_{\text {Micro }} / \mathrm{cm}^{3} \cdot \mathrm{g}^{-1}$ & $\mathrm{~V}_{\text {Meso }} / \mathrm{cm}^{3} \cdot \mathrm{g}^{-1}$ & $\mathrm{~V}_{\mathrm{T}} / \mathrm{cm}^{3} \cdot \mathrm{g}^{-1}$ \\
\hline KIT-6 & 603 & 0.02 & 0.68 & 0.76 \\
\hline $\mathrm{K} 0,012$ & 465 & 0.01 & 0.55 & 0.70 \\
\hline $\mathrm{K} 0,022$ & 572 & 0.01 & 0.63 & 0.72 \\
\hline KDT2 & 573 & 0.03 & 0.65 & 0.71 \\
\hline KDT4 & 562 & 0.01 & 0.68 & 0.73 \\
\hline KDT8 & 636 & 0.02 & 0.72 & 0.87 \\
\hline KMt & 528 & 0.01 & 0.65 & 0.70 \\
\hline KEt & 576 & 0.01 & 0.71 & 0.79 \\
\hline $\mathrm{KPr}$ & 531 & 0.01 & 0.65 & 0.75 \\
\hline KAT12 & 562 & 0.01 & 0.62 & 0.73 \\
\hline KAT18 & 462 & 0.01 & 0.56 & 0.69 \\
\hline KAT30 & 432 & 0.01 & 0.52 & 0.65 \\
\hline KHT12 & 532 & 0.02 & 0.57 & 0.67 \\
\hline KHT18 & 546 & 0.01 & 0.61 & 0.72 \\
\hline КНT30 & 624 & 0.01 & 0.72 & 0.81 \\
\hline
\end{tabular}

$\mathrm{S}_{\mathrm{BET}}=\overline{\text { specific area; } \mathrm{V}_{\mathrm{T}}=\text { total pore volume; } \mathrm{V}_{\text {Meso }}=\text { mesopores volume; } \mathrm{V}_{\text {Micro }}=\text { micropores volume }}$. 
The discussions of the textural properties were approached first for the parameters of specific area $\left(\mathrm{S}_{\mathrm{BET}}\right)$, microporous volume, mesoporous volume $\left(\mathrm{V}_{\mathrm{MESO}}\right)$ and total pore volume $\left(\mathrm{V}_{\mathrm{T}}\right)$, and later for the PSD, with the parameters average pore diameter and wall thickness $\left(\mathrm{W}_{\mathrm{T}}\right)$.

The results obtained in the modification on molar proportion of organic template reveals that the reduction of P123 concentration (K0.012 sample) promoted a decrease of more than $130 \mathrm{~m}^{2} \cdot \mathrm{g}^{-1}$ in specific area, and $0.13 \mathrm{~cm}^{3} \cdot \mathrm{g}^{-1}$ in the values of the mesoporous volume. These variations can be explained by the decrease of micelles formed in the initial phase of the synthesis, reducing the interaction of the $\mathrm{Si}-\mathrm{OH}$ groups with the organic phase. As a consequence, there was a restriction in the quantity of channels formed, resulting in a lower value of mesoporous volume, when compared with the standard KIT- 6 material, since both have 3D cubic structure. The significant value of total volume for the K0.012 sample can be explained by the adsorbed volume at pressures above $0.8 \mathrm{P} / \mathrm{P}_{0}$, which volume can be attributed to the interparticle spaces. For the K0.022 sample, it denotes that the increase in concentration of $\mathrm{P} 123$ provided the formation of a material with unidirectional hexagonal structure like as SBA-15, with relevant textural properties and similar to materials described in the literature $[39,40]$

Analyzing the results of the modification on the dissolution time of P123 for the materials with $3 \mathrm{D}$ cubic structure, it could be noted that a remarkable a proportional increase in the specific area occurred as the time increases (KDT4, KIT-6 and KDT-8 samples, respectively). This proportional difference can be explained by the dissolution time of $\mathrm{P} 123$, which is necessary for the organic-inorganic phase interactions to occur in a more efficient way, reflecting in the specific area. The data for the mesoporous volume and total volume of the KDT8 sample showed a significant increase when compared to the standard KIT-6, while for the KDT4 sample such values were similar (see Table 2). Such results complement the discussions on the X-ray diffractogram, where it can be determined that the increase on micellar formation positively influences the textural properties of the materials obtained. Both materials had textural properties similar to those found in the literature [5,41]. Analyzing the data for the KDT2 sample, relative to the unidirectional hexagonal structure of the SBA-15 type, relevant textural properties emphasizing the high mesoporous and microporous volume can be noted.

The modification of the type of alcohol promoted some changes in the textural properties of the materials, besides the targeting of the unidirectional hexagonal structure already described in the XRD analysis. This denotes a growing behavior of the specific area and the total and mesoporous volumes when comparing the KMt and KEt samples. However, for the sample synthesized with propanol (KPr sample), it denotes decreasing behavior, corroborating XRD observations and fortifying the idea that the size of the carbonic chain directly interferes on the synthesis mechanism. It is possible to note through these results that the disorder seen in the XRD analysis for the KPr sample can also be observed in the textural properties, which can indicate that this material shows a structure with disorderly channels. However, it should be emphasized that, even if the desired cubic materials were not obtained, the results are quite interesting. Highly organized mesoporous materials of SBA-15 type were obtained, with relevant textural properties and with a reduction of $24 \mathrm{~h}$ in synthesis time, when compared to the typical synthesis of these materials [28]. This denotes the quality of the material obtained, when comparing the samples in question with some found in the literature, like those reported by Kruk et al. [33] whereby the author synthesized materials of SBA-15 type with pore diameters between 5.8 and $7.5 \mathrm{~nm}$ and pore volumes between 0.4 and $0.8 \mathrm{~cm}^{3} \cdot \mathrm{g}^{-1}$.

From the materials obtained through the modification of aging time in the synthesis gel (KAT12, KAT18 and KAT30 samples), it was observed that the variation in time significantly affected the textural properties. When evaluating the materials with $3 \mathrm{D}$ cubic structure, the values of specific area $\left(\mathrm{S}_{\mathrm{BET}}\right)$, mesoporous volume $\left(\mathrm{V}_{\mathrm{MESO}}\right)$ and total volume $\left(\mathrm{V}_{\mathrm{T}}\right)$ were lower (see Table 2$)$ for both $18 \mathrm{~h}$ and $30 \mathrm{~h}$ when compared to the $24 \mathrm{~h}$ for the standard KIT-6. In this case, the aging time defines an optimal equilibrium point of $24 \mathrm{~h}$ to promote the hydrolysis of the silica source (TEOS), and later condensation of the inorganic phase interacting with the organic phase, generating exceptional textural properties for the 3D cubic structure. 
It can be observed that the variation in the time of the thermal treatment significantly influences the textural properties of the samples (KHT12, KHT18 and KHT30), and keeps the target for the 3D cubic structure. It denotes the proportional growth of values for the $S_{B E T}, V_{M E S O}$ and $V_{T}$ parameters when the thermal treatment time increases (see Table 2). This behavior occurs because the longer the treatment time the greater the condensation rate of the silanol groups $(\mathrm{Si}-\mathrm{OH})$, directly influencing the formation of the inorganic structure.

All the materials presented microporous volume varying between 0.01 and $0.03 \mathrm{~cm}^{3} \cdot \mathrm{g}^{-1}$. In some specific cases, a small difference in the microporous volume is observed, such as in the case of the KHT12, KHT18 and KHT30 samples. However, the $\alpha$-plot method does not allow a more in-depth analysis of these data, requiring more efficient methods, such as $\mathrm{CO}_{2}$ adsorption at high pressures [42].

Table 4 shows the values of average pore diameter calculated with BJH, NLDFT and with VBS from the adsorption branch to compare the methods each other, and the values of pore diameter calculated from the desorption branch calculated by the VBS method. Figure 5 representatively shows the PSD of the standard KIT-6 and KDT2 samples.

Table 4. Average values of pore diameter through the Barrett-Joyner-Halanda (BJH), Villarroel-Bezerra-Sapag (VBS) and non-local density functional theory (NLDFT) techniques of all samples with ordered structures.

\begin{tabular}{|c|c|c|c|c|c|}
\hline Samples & $\mathrm{W}_{\mathrm{BJH}} / \mathrm{nm}$ & $\mathrm{W}_{\mathrm{VBS}} / \mathbf{n m}$ & $\mathrm{W}_{\mathrm{NLDFT}} / \mathrm{nm}$ & $\mathrm{W}_{\mathrm{VBS}} / \mathrm{nm}$ & \multirow{2}{*}{$\mathrm{Wt} / \mathrm{nm}$} \\
\hline & & Adsorption & & Desorption & \\
\hline KIT-6 & 7.2 & 6.8 & 9.6 & 7.0 & 5.8 \\
\hline K0.012 & 6.8 & 7.0 & 9.1 & 6.6 & 6.9 \\
\hline K0.022 & 6.6 & 6.8 & 8.7 & 6.7 & 5.7 \\
\hline KDT2 & 6.7 & 6.8 & 9.1 & 6.6 & 6.1 \\
\hline KDT4 & 6.9 & 7.0 & 9.2 & 6.5 & 4.6 \\
\hline KDT8 & 7.2 & 7.2 & 9.6 & 7.2 & 5.4 \\
\hline KMt & 6.5 & 6.7 & 8.7 & 6.5 & 6.2 \\
\hline $\mathrm{KEt}$ & 6.8 & 7.0 & 9.1 & 6.7 & 6.5 \\
\hline $\mathrm{KPr}$ & 6.5 & 6.7 & 8.9 & 6.7 & 5.0 \\
\hline KAT12 & 6.7 & 6.7 & 8.3 & 6.6 & 5.7 \\
\hline KAT18 & 6.9 & 7.3 & 8.2 & 7.5 & 5.2 \\
\hline КАТ30 & 6.0 & 7.5 & 8.2 & 7.3 & 5.6 \\
\hline KHT12 & 6.1 & 6.5 & 8.2 & 6.6 & 5.9 \\
\hline KHT18 & 6.2 & 6.6 & 8.3 & 6.8 & 5.8 \\
\hline КНТ30 & 7.0 & 6.9 & 9.0 & 7.0 & 5.6 \\
\hline
\end{tabular}

$\mathrm{W}_{\mathrm{BJH}}=$ pore diameter using the BJH method. $\mathrm{W}_{\mathrm{NLDFT}}=$ pore diameter using the NLDFT method. $\mathrm{W}_{\mathrm{VBS}}=$ pore diameter using the VBS method. $\mathrm{Wt}=$ wall thickness calculated from: $\mathrm{a}_{\mathrm{Cub}}-\mathrm{W}_{\mathrm{VBS}}$ (cubic structures) and $\mathrm{a}_{\mathrm{Hex}}-\mathrm{W}_{\mathrm{VBS}}$ (hexagonal structures).

The PSD is very important to define the pore volumes for each pore size and, thus, to obtain the values of the average pore diameter of nanoporous materials. From the $\mathrm{N}_{2}$ adsorption-desorption experimental isotherm data at $77 \mathrm{~K}$, it was possible to apply microscopic (based on molecular theories) and macroscopic (based on capillary condensation) methods. According to some [24,43,44], the most used microscopic method is the NLDFT, and the macroscopic methods are BJH and VBS, these last two based on the Kelvin equation.

In this work, the materials obtained with cylindrical pores show reversible isotherms with filling/emptying mechanism and hysteresis loops that are well defined. For this reason, the adsorption branch isotherm was selected to compare the methods with each other. From the evaluation of the PSD of representative samples (Figure 6) and the values of average pore diameter of all samples (Table 4), the NLDFT model overestimated the value of the pore size, while the values of the BJH and VBS methods were very close. This behavior suggests that the NLDFT model was not applied efficiently, once it takes another predetermined kernel to best fit the behavior of the samples. Among the macroscopic methods, the VBS method is the most accepted because this method was improved by 
adding a correction term to the original Kelvin equation, providing more accurate values for the pore size. Taking this into account, all discussion about the PSD and average pore diameter were carried out based on the VBS method applied in the desorption branch. This is because, according to the authors [24,44], the desorption branch reflects the transition of the equilibrium phase, which supports the macroscopic methods that use the Kelvin equation.

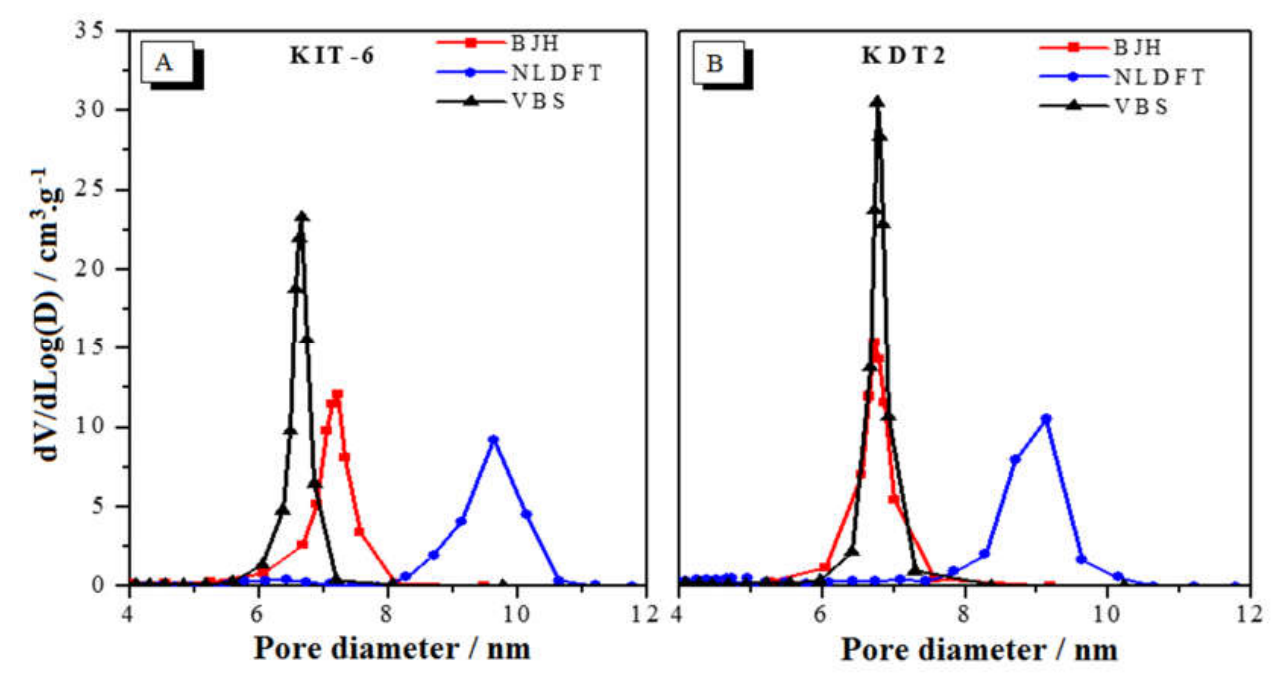

Figure 6. Pore size distribution (PSD) of the (A) standard KIT-6 and (B) KDT2 samples.

From the data of the K0.012 and K0.022 samples obtained, there can be seen a similarity in the values of pore diameter when compared to the standard KIT- 6 . Such a fact demonstrates that the variation of the amount of organic template did not influence in an expressive way the pore size of the materials. However, when analyzing the values of wall thickness, a difference of $1.1 \mathrm{~nm}$ between the K0.012 sample and the standard KIT- 6 can be noted. This difference is directly related to the values of cubic parameters observed (see Table 2). In consequence, such behavior is reflected in the wall thickness. Thus, the wall thickness values contribute to the explanation of the synthesis mechanism and the specific area values for such materials, as cited previously.

Evaluating the dissolution time for the materials with 3D cubic structures, the KDT4 and KDT8 samples showed proportional differences for pore diameter, when compared to the standard KIT-6. The values of pore diameter exhibited a difference of $0.4 \mathrm{~nm}$ (values of 6.5, 6.9 and $7.3 \mathrm{~nm}$ ) between the KDT4, KIT-6 and KDT8 samples, respectively. This behavior indicates an increase in the average pore size in function of the dissolution time of the organic template. Analyzing the values of wall thickness, it denotes a significant decrease of $1.2 \mathrm{~nm}$ on the KDT4 sample, when compared to the standard KIT-6. This data correlates the values observed for the cubic parameter $\left(\mathrm{a}_{\mathrm{cub}}\right)$ and, in consequence, specific area $\left(\mathrm{S}_{\mathrm{BET}}\right)$, corroborating with the synthesis mechanism cited. The KDT8 sample was compared with the standard KIT- 6 and it is worth mentioning that, despite the values of wall thickness being similar, the KDT8 sample demonstrated superior textural properties (specific area, mesoporous volume, total volume and pore diameter) with the addition of $2 \mathrm{~h}$ in the synthesis process.

Evaluating the pore diameter data for the KMt, KEt and KPr materials with hexagonal structure, this shows very similar behavior for each one. On the other hand, a very irregular behavior was seen when comparing the data obtained for wall thickness of these materials. A similarity between the KMt and KEt samples (6.2 and $6.5 \mathrm{~nm}$, respectively) can be noted, while the KPr sample shows a significantly inferior value (about $1.3 \mathrm{~nm}$ ). This behavior relates to the values of interplanar distance and mesoporous parameter for the unidirectional hexagonal structure. This set of facts reveals that the 
type of alcohol used in the synthesis acts only as a co-solvent for the formation of the SBA-15 structure, slightly modifying the textural properties, but not significantly interfering in the micellar formation.

The samples that displayed 3D cubic structure in the modification of aging time of the synthesis gel (KAT18 and KAT30 samples) presented values of average pore diameter and wall thickness similar to the standard KIT-6 material. Such a result suggests that this synthesis parameter does not significantly interfere in the formation and micellar organization. However, as discussed earlier, there is an optimal equilibrium time of $24 \mathrm{~h}$ to promote better textural properties values $\left(\mathrm{S}_{\mathrm{BET}}, \mathrm{V}_{\mathrm{MESO}}\right.$ and $\left.\mathrm{V}_{\mathrm{T}}\right)$, based in the stages of hydrolysis of the silica and condensation of the inorganic phase. From this, it is of great relevance to point out that the aging time of synthesis gel favors the 3D cubic structure for the KIT-6 material starting from $18 \mathrm{~h}$ of procedure, reaching the best textural properties in $24 \mathrm{~h}$.

Analyzing the samples obtained in the variation of thermal treatment time, this denotes a small increase in the average pore diameter $(0.4 \mathrm{~nm})$ and a slight decrease in wall thickness $(0.3 \mathrm{~nm})$, when comparing the shortest time studied (KHT12 sample) with the longest (KHT30 sample). These results reveal that the modification of thermal treatment time, in which occurs the condensation of the silanol groups, does not significantly influence the micellar formation and consequently the average pore diameter. However, the thermal treatment time acts considerably in the synthesis mechanism, increasing the values of the textural properties (specific area, mesoporous volume and total volume), when compared to the 12 and $30 \mathrm{~h}$ times (see Table 2). Nevertheless, it is important to emphasize that, starting with $12 \mathrm{~h}$ of thermal treatment, the 3D cubic structure is formed with relevant textural properties. With an increase in $6 \mathrm{~h}$ at a time between the KIT-6 and KHT30 samples, the 3D cubic structure reflects similar textural properties.

Figure 7 exhibits TEM micrographs of KDT4 and KHT12 representative samples.
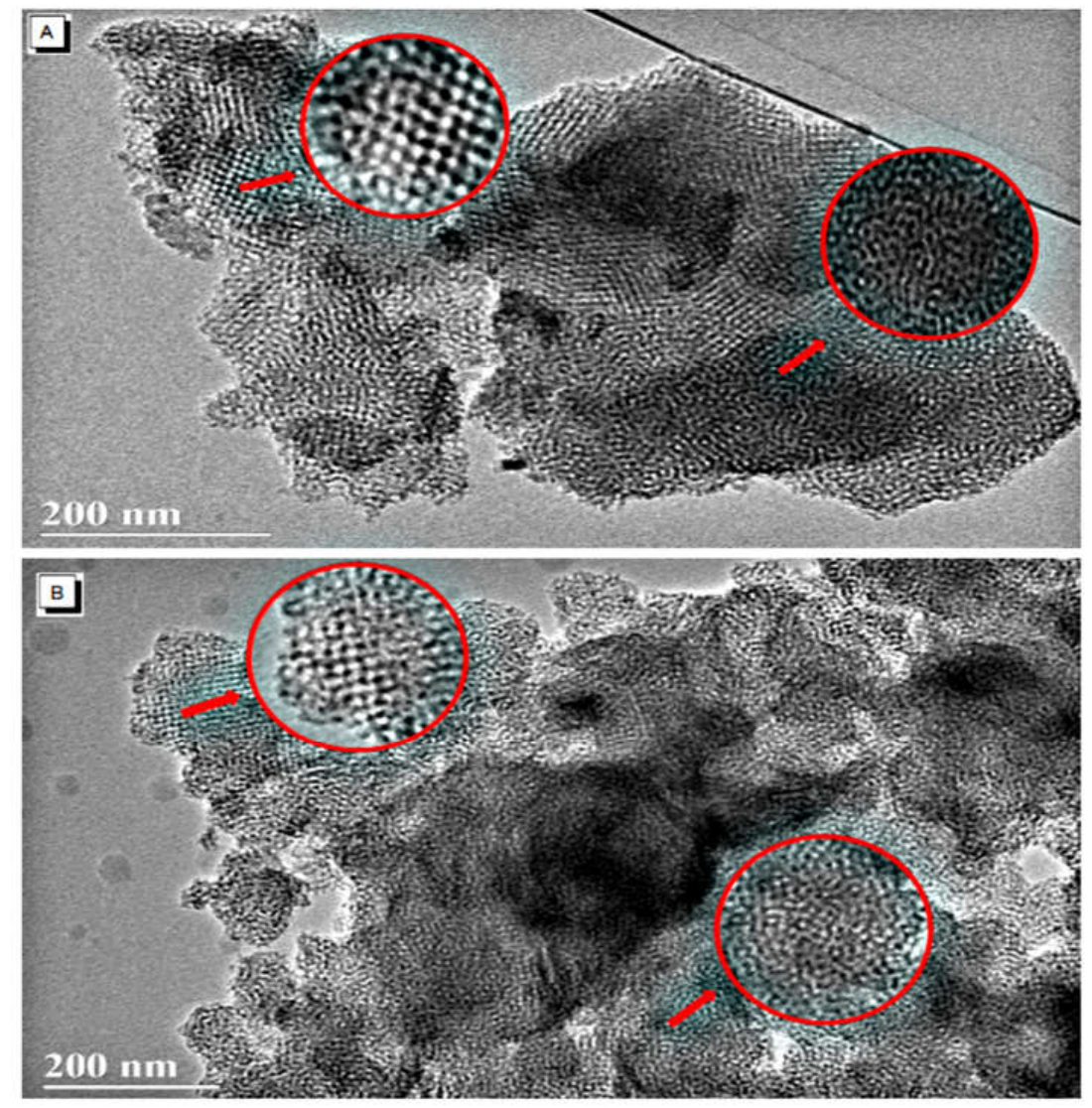

Figure 7. Representative transmission electron microscope (TEM) images of (A) KDT4 and (B) KHT12 samples. 
The images of the KDT4 and KHT12 samples are presented in a representative way for the materials that showed a 3D cubic structure. Analyzing the TEM micrographies of these materials, it was observed along the axis [001] that the two samples showed well-defined pore organization. When analyzing the image along the axis [010], it can be clearly noticed that the channel curvatures refer to the type of bi-continuous channels respective to KIT- 6 mesoporous materials. Such behavior can also be visualized in studies described in the literature $[9,45]$. These results corroborate those found in the XRD technique and $\mathrm{N}_{2}$ adsorption-desorption isotherms.

Through this technique, the pore diameter of these materials was also measured. The KDT4 and KHT12 samples presented average values of $6.4 \pm 1.23$ and $5.9 \pm 0.95 \mathrm{~nm}$ for the pore diameters, respectively. These values are very similar to those found in the pore size distribution (PSD), calculated by the VBS method in the desorption branch through $\mathrm{N}_{2}$ adsorption-desorption isotherms. From this, it is important to emphasize two points: the employment of the VBS method for measuring the pore diameter by the pore size distribution (PSD) was precisely appropriated; and the modification in some parameters, reducing the time at synthesis procedure, promoted KIT-6 materials with a well-defined 3D cubic structure.

\section{Conclusions}

The KIT-6 mesoporous material have been efficiently synthesized through a typical procedure, reported in the literature. Based on this procedure, modifications to the synthesis parameters have been presented and show a significant influence on the structure and textural properties in the ordered mesoporous materials obtained. For the formation of Ia3d cubic structures, characteristic of the KIT-6 material, concentrations of P123 below $0.017 \mathrm{M}$ are required. The alcohol probably acts on the generation of the micelle, and the size of its carbonic chain possibly interferes in the formation of the porous system and ordered structure of the synthesized mesoporous materials. In order to obtain the 3D cubic structure, a longer synthesis time is required, otherwise SBA-15 or any ordered structures are formed. In other words, below $4 \mathrm{~h}$ and $18 \mathrm{~h}$ for P123 dissolution and aging gel, respectively, the SBA-15 ordered structure is generated. The heat treatment time has a significant influence on the textural properties of the synthesized materials. Thus, this parameter revealed that the longer the time employed, the better the materials that are produced, by demonstrating elevated specific area, high volume and pore diameter. The data obtained have confirmed the influence of the synthesis parameters in the formation, organization and textural properties of mesoporous material like KIT-6. However, it is important to emphasize that the cubic structure of KIT- 6 can be obtained by reducing the concentration and dissolution time of P123, and times of heat treatment and aging, when compared to the synthesis reported in the literature. The modifications carried out in the synthesis procedure have resulted in materials with different characteristics, which makes it possible to employ them in applications in the areas of catalysis and adsorption.

Supplementary Materials: The following are available online at http:/ /www.mdpi.com/2076-3417/8/5/725/s1, Figure S1: Curves of (A) TG and (B) DTG of samples with cubic structures, Figure S2: Curves of (A) TG and (B) DTG of samples with hexagonal structures, Figure S3: FTIR spectra of all samples with ordered structures.

Author Contributions: F.R.D.F. and E.L.F.L. performed the experiments. F.R.D.F., F.G.H.S.P., A.G.D.S. and V.P.S.C. wrote the paper and analyzed the data. L.D.S., V.P.S.C. and A.G.D.S. supervised the whole work.

Acknowledgments: The authors thanks to the CAPES/Brazil for the financial support, and the Magnetic and Optical Analysis Laboratory (LAMOp/State University of Rio Grande do Norte), and GERATEC-PPGQ-CCN/State University of Piauí, for the analysis performed.

Conflicts of Interest: The authors declare no conflict of interest. The founding sponsors had no role in the design of the study; in the collection, analyses, or interpretation of data; in the writing of the manuscript, and in the decision to publish the results. 


\section{References}

1. Prabhu, A.; Kumaresan, L.; Palanichamy, M.; Murugesan, V. Synthesis and characterization of aluminium incorporated mesoporous KIT-6: Efficient catalyst for acylation of phenol. Appl. Catal. A Gen. 2009, 360, 59-65. [CrossRef]

2. Qian, L.; Ren, Y.; Liu, T.; Pan, D.; Wang, H.; Chen, G. Influence of KIT-6's pore structure on its surface properties evaluated by inverse gas chromatography. Chem. Eng. J. 2009, 213, 186-194. [CrossRef]

3. Kalsabi, R.J.; Mosaddegh, N. Pd-poly(N-vinyl-2-pyrrolidone)/KIT-6 nanocomposite: Preparation, structural study, and catalytic activity. C. R. Chim. 2012, 15, 988-995. [CrossRef]

4. Dou, B.; Hu, Q.; Li, J.; Qiao, S.; Hao, Z. Adsorption performance of VOCs in ordered mesoporous silicas with different pore structures and surface chemistry. J. Hazard. Mater. 2011, 186, 1615-1624. [CrossRef] [PubMed]

5. Boulaoued, A.; Fechete, I.; Donnio, B.; Bernard, M.; Turek, P.; Garin, F. Mo/KIT-6, Fe/KIT-6 and Mo-Fe/KIT-6 as new types of heterogeneous catalysts for the conversion of MCP. Microporous Mesoporous Mater. 2012, 155, 131-142. [CrossRef]

6. Kumaseran, L.; Prabhu, A.; Palanichamy, M.; Murugesan, V. Mesoporous Ti-KIT-6 molecular sieves: Their catalytic activity in the epoxidation of cyclohexene. J. Taiwan Inst. Chem. Eng. 2010, 41, 670-675. [CrossRef]

7. Falahati, M.; Ma'mani, L.; Saboury, A.A.; Shafiee, A.; Foroumadi, A.; Badiei, A.B. Aminopropyl-functionalized cubic Ia3d mesoporous silica nanoparticle as an efficient support for immobilization of superoxide dismutase. Biochim. Biophys. Acta 2012, 1814, 1195-1202. [CrossRef] [PubMed]

8. Karthikeyan, G.; Pandurangan, A. Post synthesis alumination of KIT-6 materials with Ia3d symmetry and their catalytic efficiency towards multicomponent synthesis of $1 H$-pyrazolo [1,2-]phthalazine-5,10-dione carbonitriles and carboxylates. J. Mol. Catal. A Chem. 2012, 361, 58-67. [CrossRef]

9. Kleitz, F.; Choi, S.H.; Ryoo, R. Cubic Ia3d mesoporous sílica: Synthesis and replication to platinum nanowires, carbon nanorods and carbon nanotubes. Chem. Commun. 2003, 17, 2136-2137. [CrossRef]

10. Zhao, Y.; Chen, X.; Yang, C.; Zhang, G. Mesoscopic Simulation on Phase Behavior of Pluronic P123 Aqueous Solution. J. Phys. Chem. B 2007, 111, 13937-13942. [CrossRef] [PubMed]

11. Wang, Y.; Zhang, F.; Wang, Y.; Ren, J.; Li, C.; Liu, X.; Guo, Y.; Guo, Y.; Lu, G. Synthesis of length controllable mesoporous SBA-15 rods. Mater. Chem. Phys. 2009, 115, 649-655. [CrossRef]

12. Impéror-Clerc, M.; Manet, S.; Grillo, I.; Durand, D.; Khodakov, A.; Zholobenko, V. Study of the mechanisms and kinetics of the synthesis of mesoporous materials from micelles of tri-block copolymers. Stud. Surf. Sci. Catal. 2008, 174, 805-810. [CrossRef]

13. Guan, L.S.; Nur, H.; Endud, S. Bimodal pore size mesoporous mcm-48 prepared by post-synthesis alumination. J. Phys. Sci. 2006, 17, 65-75.

14. Chareonpanich, M.; Nanta-Ngern, A.; Limtrakul, J. Short-period synthesis of ordered mesoporous silica SBA-15 using ultrasonic technique. Mater. Lett. 2007, 61, 5153-5156. [CrossRef]

15. Cao, L.; Dong, H.; Huang, L.; Matyjaszewski, K.; Kruk, M. Synthesis of large pore SBA-15 silica using poly(ethylene oxide)-poly(methyl acrylate) diblock copolymers. Adsorption 2009, 15, 156-166. [CrossRef]

16. Galarneau, A.; Cambon, H.; Renzo, F.D.; Ryoo, R.; Choi, M.; Fajula, F. Microporosity and connections between pores in SBA-15 mesostructured silicas as a function of the temperature of synthesis. New J. Chem. 2002, 7, 73-79. [CrossRef]

17. Kang, K.; Rhee, H. Synthesis and characterization of novel mesoporous silica with large wormhole-like pores: Use of TBOS as silicon source. Microporous Mesoporous Mater. 2005, 84, 34-40. [CrossRef]

18. Iglesias, J.; Melero, J.A.; Sainz-pardo, J. Direct synthesis of organically modified Ti-SBA-15 materials. J. Mol. Catal. A Chem. 2008, 291, 75-84. [CrossRef]

19. Qiang, Z.; Gurkan, B.; Ma, J.; Liu, X.; Guo, Y.; Cakmak, M.; Cavicchi, K.A.; Vogt, B.D. Roll-to-roll fabrication of high surface area mesoporous carbon with process-tunable pore texture for optimization of adsorption capacity of bulky organic dyes. Microporous Mesoporous Mater. 2016, 227, 57-64. [CrossRef]

20. Deng, G.; Zhang, Y.; Ye, C.; Qiang, Z.; Stein, G.E.; Cavicchi, K.A.; Vogt, B.D. Bicontinuous mesoporous carbon thin films via an order-order transition. Chem. Commun. 2014, 50, 12684-12687. [CrossRef] [PubMed]

21. Brunauer, B.S.; Emment, P.H.; Teller, E. Adsorption of gases in multimolecular layers. J. Am. Chem. Soc. 1938, 60, 309-319. [CrossRef] 
22. Rouquerol, J.; Llewellyn, P.; Rouquerol, F. Is BET equation applicable to microporous adsorbents? Stud. Surf. Sci. Catal. 2007, 160, 49-56.

23. Rouquerol, F.; Rouquerol, J.; Sing, K.S.W.; Llewellyn, P.; Maurin, G. Adsorption by Powders and Porous Solids, 2nd ed.; Methodology and Applications, Academic Press: San Diego, CA, USA, 2014; ISBN 978-0-08-097035-6.

24. Villarroel-Rocha, J.; Barrera, D.; Sapag, K. Introducing a self-consistent test and the corresponding modification in the Barrett, Joyner and Halenda method for pore-size determination. Microporous Mesoporous Mater. 2014, 200, 68-78. [CrossRef]

25. Zukal, A.; Thommes, M.; Čejka, J. Synthesis of highly ordered MCM-41 silica with spherical particles. Microporous Mesoporous Mater. 2007, 104, 52-58. [CrossRef]

26. He, C.; Li, J.; Zhang, X.; Yin, L.; Chen, J.; Gao, S. Highly active Pd-based catalysts with hierarchical pore structure for toluene oxidation: Catalyst property and reaction determining factor. Chem. Eng. J. 2012, 180, 46-56. [CrossRef]

27. Zhao, H.; Liu, S.; Wang, R.; Zhang, T. Humidity-sensing properties of LiCl-loaded 3D cubic mesoporous silica KIT-6 composites. Mater. Lett. 2015, 147, 54-57. [CrossRef]

28. Zhao, D.; Feng, J.; Huo, Q.; Melosh, N.; Fredrickson, G.H.; Chmelka, B.F.; Stucky, G.D. Triblock Copolymer Syntheses of Mesoporous Silica with Periodic 50 to 300 Angstrom Pores. Science 1998, 279, 548-552. [CrossRef] [PubMed]

29. Wanka, G.; Hoffmann, H.; Ulbricht, W. Phase Diagrams and Aggregation Behavior of Poly(oxyethylene)Poly(oxypropylene)-Poly(oxyethylene) Triblock Copolymers in Aqueous Solutions. Macromolecules 1994, 27, 4145-4159. [CrossRef]

30. Kim, T.W.; Kleitz, F.; Paul, B.; Ryoo, R. MCM-48-like Large Mesoporous Silicas with Tailored Pore Structure: Facile Synthesis Domain in a Ternary Triblock Copolymer-Butanol-Water System. J. Am. Chem. Soc. 2005, 127, 7601-7610. [CrossRef] [PubMed]

31. Kim, J.M.; Ryoo, R. Synthesis of MCM-48 single crystals. Chem. Commun. 1998, 2, 259-260. [CrossRef]

32. Liu, C.; Wang, X.; Lee, S.; Pfefferle, L.D.; Haller, G.L. Surfactant chain length effect on the hexagonal-to-cubic phase transition in mesoporous silica synthesis. Microporous Mesoporous Mater. 2012, 147, 242-251. [CrossRef]

33. Kruk, M.; Jeroniec, M.; Ko, C.H.; Ryoo, T. Characterization of the Porous Structure of SBA-15. Chem. Mater. 2000, 12, 1961-1968. [CrossRef]

34. Fernandes, F.R.D.; Santos, A.G.D.; Souza, L.D.; Santos, A.P.B. Síntese e caracterização do material mesoporoso sba-15 obtido com diferentes condições de síntese. Rev. Virtual Química 2016, 8, 1855-1864. [CrossRef]

35. Shukla, P.; Sun, H.; Wang, S.; Ang, H.M.; Tadé, M.O. Co-SBA-15 for heterogeneous oxidation of phenol with sulfate radical for wastewater treatment. Catal. Today 2011, 175, 380-385. [CrossRef]

36. Wang, X.Q.; Ge, H.L.; Jin, H.X.; Cui, Y.J. Influence of Fe on the thermal stability and catalysis of SBA-15 mesoporous molecular sieves. Microporous Mesoporous Mater. 2005, 86, 335-340. [CrossRef]

37. Guo, Y.H.; Xia, C.; Liu, B.S. Catalytic properties and stability of cubic mesoporous LaxNiyOz/KIT-6 catalysts for $\mathrm{CO} 2$ reforming of $\mathrm{CH} 4$. Chem. Eng. J. 2014, 237, 421-429. [CrossRef]

38. Thommes, M.; Koneko, K.; Neimark, A.V.; Oliver, J.P.; Rodirguez-reinoso, F.; Rouquerol, J.; Sing, K.S.W. Physisorption of gases, with special reference to the evaluation of surface area and pore size distribution (IUPAC Technical Report). Pure Appl. Chem. 2015, 87, 1051-1069. [CrossRef]

39. Wang, S.; Wang, K.; Dai, C.; Shi, H.; Li, J. Adsorption of $\mathrm{Pb}^{2+}$ on amino-functionalized core-shell magnetic mesoporous SBA-15 silica composite. Chem. Eng. J. 2015, 262, 897-903. [CrossRef]

40. Vizcaíno, A.J.; Carrero, A.; Calles, J.A. Comparison of ethanol steam reforming using Co and Ni catalysts supported on SBA-15 modified by Ca and Mg. Fuel Process. Technol. 2016, 146, 99-109. [CrossRef]

41. Hussain, M.; Akhter, P.; Russo, N.; Saracco, G. Novel Ti-KIT-6 material for the photocatalytic reduction of carbon dioxide to methane. Catal. Commun. 2013, 36, 58-62. [CrossRef]

42. Thommes, M.; Chychosz, K.A.; Neimark, A.V. Advanced physical adsorption characterization of nanoporous carbons. Novel Carb. Adsorbents 2012, 107-139. [CrossRef]

43. Neimark, A.V.; Ravikovitch, P.I. Density Functional Theory of Adsorption Hysteresis and Nanopore Characterization. Stud. Surf. Sci. Catal. 2000, 128, 51-60. [CrossRef] 
44. Rocha, J.V.; Barrera, D.; Sapag, K. Improvement in the Pore Size Distribution for Ordered Mesoporous Materials with Cylindrical and Spherical Pores Using the Kelvin Equation. Top. Catal. 2011, 54, 121-134. [CrossRef]

45. Wang, J.; Li, Y.; Zhang, Z.; Hao, Z. Mesoporous KIT-6 silica-polydimethylsiloxane (PDMS) mixed matrix membranes for gas separation. J. Mater. Chem. A 2015, 3, 8650-8658. [CrossRef] 